\title{
Narrow-band imaging and a search for planetary nebulae ${ }^{\star}$
}

\author{
T. Rauch ${ }^{\star \star}$ \\ Institut für Astronomie und Astrophysik der Universität, D-72076 Tübingen, Germany
}

Received October 2; accepted November 12, 1998

\begin{abstract}
We summarize the results of narrow-band ( $\mathrm{H} \alpha$ and [O III $] \lambda 5007 \AA$ ) imaging of eight planetary nebulae taken during recent observing campaigns: We present the probably largest known PN, MWP 1, with a linear diameter of about $6 \mathrm{pc}$. We show first narrow-band images of He 2-437, MeWe 1-5, MeWe 1-8, MeWe 1-9, NGC 1360, and of - like we term them - the "Galactic footballs" A 43 and NGC 7094.

We report about a search for planetary nebulae around eleven extremely hot (pre-) white dwarfs which had not been successful.
\end{abstract}

Key words: planetary nebulae: general — planetary nebulae: individual: A 43; He 2-437; MWP 1; NGC 1360; NGC 7094

\section{Introduction}

Planetary nebulae $(\mathrm{PN})$ and their central stars (CSPN) are ideal tools to check against evolutionary theory: After the ejection of the PN still on the Asymptotic Giant Branch (AGB), the effective temperature $\left(T_{\text {eff }}\right)$ of the exciting star increases at almost constant luminosity. The stars can reach very high $T_{\text {eff }}(>100 \mathrm{kK}$, e.g. Rauch \& Werner 1998). At their hottest stage of evolution, these post-AGB stars are found at the beginning of the white dwarf (WD) cooling sequence where the nuclear burning is close to its end and gravitation is beginning to dominate the further evolution.

Send offprint requests to: T. Rauch

* Based on observations obtained at the German-Spanish Astronomical Center, Calar Alto, operated by the Max-PlanckInstitut für Astronomie Heidelberg jointly with the Spanish National Commission for Astronomy and at the European Southern Observatory, La Silla, Chile.

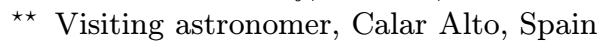

Correspondence to: rauch@astro.uni-tuebingen.de
Modern spectral analysis of CSPN (e.g. Rauch et al. 1998) provides photospheric parameters which are stringent constraints for evolutionary theory.

The development of both, observational as well as numerical methods, provide now high-resolution images of PN (e.g. Balick et al. 1998) and deprojection techniques (Bremer 1995) which improve the interpretation of the nebula morphology. This will enable us to construct consistent models of PN and central star: Rauch et al. (1994, 1996) have successfully used synthetic ionizing spectra instead of the commonly used blackbodies in order to improve the photoionization model of the nebula. The derived spectroscopic distances allow to calculate the linear dimensions of the PN. Recent improvements of the interacting stellar wind model which take into account timedependent winds (Dwarkadas \& Balick 1998) have shown for the first time the possibility to model small-scale structures which can be observed in high-resolution images. As a by-product, the expansion times of the PN can be determined more reliably and give a much better estimate of the time since the central star has departed from the AGB. Moreover, additional information becomes available because the long recombination time $\left(\approx 300000 \mathrm{a} / n_{\mathrm{e}}\right)$ in these low density PN $\left(n_{\mathrm{e}}<100 \mathrm{~cm}^{-3}\right)$ allows a view onto the emission phase of the nebula, e.g. the photospheric abundances at that time providing a second check for theoretical predictions of stellar evolution.

Since the PNe contribute to the metal enrichment of the interstellar medium (ISM), they play also an important role in the galactic chemical evolution. Studies of the interaction of aging PN with the ISM by hydrodynamical simulations (e.g. Soker \& Dgani 1997) which aim to describe the complex interaction zone need, as a prerequisite, reliable information about nebula properties and its spatial distribution of matter.

During recent observing campaigns, we took narrowband images of several PN. In the following sections we present eight of them which exhibit interesting details of nebula morphology and allow to judge the degree of interaction with the ISM. 
Table 1. List of our observed PN. The field sizes (fs) of the images shown as well as the measured angular nebula diameters (faintest visible part of the main nebula on the $\mathrm{H} \alpha$ images) $d$ in West-East $\left(d_{\mathrm{WE}}\right)$ and North-South direction $\left(d_{\mathrm{NS}}\right)$ are given. *: For the elliptical nebulae MWP 1 and NGC 1360 the diameters are given for their axis in NW-SE/SE-NW direction. The exposure times ( $t$ in sec) were the same for both the $\mathrm{H} \alpha$ as well as the [O III] $\lambda 5007 \AA$ images. In the case of A 43 two $\mathrm{H} \alpha$ images were taken and co-added. The figure numbers $(F)$ refer to the figures in this paper. All PNe are shown with North up and East left on a linear intensity scale

\begin{tabular}{lllrrrr}
\hline PN G & name & $F$ & \multicolumn{1}{c}{ fs } & \multicolumn{1}{c}{$d_{\mathrm{WE}}$} & \multicolumn{1}{c}{$d_{\mathrm{NS}}$} & \multicolumn{1}{c}{$t$} \\
\hline $036.0+17.6$ & A 43 & 5 & $2^{\prime}$ & $1^{\prime} 28^{\prime \prime}$ & $1^{\prime} 20^{\prime \prime}$ & 1800 \\
$061.3+03.6$ & He 2-437 & 7 & $40^{\prime \prime}$ & $38^{\prime \prime}$ & $8^{\prime \prime}$ & 1800 \\
$066.7-28.2$ & NGC 7094 & 6 & $2^{\prime}$ & $1^{\prime} 45^{\prime \prime}$ & $1^{\prime} 46^{\prime \prime}$ & 1800 \\
$080.3-10.4$ & MWP 1 & 1 & $20^{\prime}$ & $14^{\prime} 45^{\prime \prime}$ & $8^{\prime} 36^{\prime \prime}$ & 1200 \\
$220.3-53.9$ & NGC 1360 & 8 & $12^{\prime}$ & $7^{\prime} 30^{\prime \prime}$ & $6^{\prime} 00^{\prime \prime}$ & 1800 \\
$321.0+08.3$ & MeWe 1-5 & 2 & $40^{\prime \prime}$ & $27^{\prime \prime}$ & $28^{\prime \prime}$ & 1800 \\
$335.6-04.0$ & MeWe 1-8 & 3 & $40^{\prime \prime}$ & $24^{\prime \prime}$ & $24^{\prime \prime}$ & 900 \\
$337.6-04.2$ & MeWe 1-9 & 4 & $40^{\prime \prime}$ & $22^{\prime \prime}$ & $21^{\prime \prime}$ & 900 \\
\hline
\end{tabular}

In the same region of the $g-T_{\text {eff }}$ plane occupied by the CSPN there are many stars for which no associated PN is known (e.g. Werner et al. 1997). This is still an unsolved problem (Rauch et al. 1998) and an outstanding challenge for evolutionary theory. However, one possible explanation for the "missing" PN might be that they have already dispersed below the detection limit of the former observations. A search for PN around hot post-AGB stars is described in the following.

\section{Observations and data reduction}

Narrow-band images ( $\mathrm{H} \alpha$ and $[\mathrm{O}$ III $] \lambda 5007 \AA$ ) of eight PN (Table 1) have been taken with the $2.2 \mathrm{~m}$ telescope and CAFOS (SITe-1d CCD, $2048 \times 204824 \mu \mathrm{m} \mathrm{px}$, $1 \mathrm{px}=0.53$ projected at the sky) at the Calar Alto observatory (only MWP 1) in August 1996 and with the $1.54 \mathrm{~m}$ Danish telescope and DFOSC (LORAL/LESSER $\mathrm{CCD}, 2052 \times 205215 \mu \mathrm{m} \mathrm{px}, 1 \mathrm{px}=0^{\prime \prime}$ 39) at ESO (ESO No. 61.C-0101) in July 1998.

A search for PN around hot (pre-) white dwarfs had been performed in October 1997 at ESO with the $1.54 \mathrm{~m}$ Danish telescope and DFOSC (ESO No. 60.C-0376). H $\alpha$ images with an exposure time of one hour had been taken. A list of observed objects is given in Table 2.

For the data reduction we used $\operatorname{IRAF}^{1}$ (Tody 1986). All images were flatfielded using suitable sky flats.

\footnotetext{
${ }^{1}$ IRAF is distributed by the National Optical Astronomy Observatories, which is operated by the Association of Universities for Research in Astronomy, Inc. (AURA) under cooperative agreement with the National Science Foundation.
}

Table 2. Targetlist for our PN search. $T_{\text {eff }}$ and $\log g$ are taken from Dreizler \& Heber (1998); Dreizler \& Werner (1996, 1997); Werner et al. $(1996,1997)$

\begin{tabular}{llcc}
\hline star & spectral type & $T_{\text {eff }} / \mathrm{kK}$ & $\log g(\mathrm{cgs})$ \\
\hline HE 0504-2408 & DO & $>80$ & \\
HS 0231+0505 & DAO & & \\
HS 0444+0453 & PG 1159 & 90 & 7.0 \\
HS 2033+0507 & DAO & & \\
HS 2115+1148 & DAO & 67 & 6.9 \\
MCT 0130-196 & PG 1159 & 90 & 7.5 \\
MCT 2148-294 & DO & 85 & 7.5 \\
PG 0046+078 & DO & 73 & 8.0 \\
PG 2131+066 & PG 1159 & 80 & 7.5 \\
RE 0503-289 & DO & 70 & 7.5 \\
RX J0122.9-7521 & PG 1159 & 180 & 7.5 \\
\hline
\end{tabular}

\section{Search for planetary nebulae around hot post-AGB stars}

A search for PN around hot (pre-) white dwarfs by means of wide-field narrow-band $\mathrm{H} \alpha \mathrm{CCD}$ imaging was performed at the northern sky (1.23 m telescope at Calar Alto, exposure times of one hour) by Werner et al. (1997). 16 objects were observed which revealed only one candidate for a PN - an asymmetric nebulosity was found around PG 0109+111.

Our search had the same aim: To find "missing" PN (Sect.1) which have not been detected so far, maybe because of their too-low surface brightness. However, no $\mathrm{H} \alpha$ emission which could stem from a nebula is detected on these images.

\section{MWP 1}

MWP 1 has been discovered by Motch et al. (1993) around one of the hottest stars ever analyzed by means of NLTE stellar atmospheres: RX J2117.1+3412, which has been discovered by ROSAT ${ }^{2}$ (Motch et al. 1991). RX J2117.1+3412 is a helium- and carbon-rich PG 1159 star with $T_{\text {eff }}=170 \mathrm{kK}$ (Werner et al. 1996). The enormous size of this PN was first noted by Appleton et al. (1993) who found an apparent diameter of $13^{\prime}$ and discuss its morphology in detail. Narrow-band images are also presented in an atlas of ancient PNe which are interacting with the ISM by Tweedy \& Kwitter (1996).

The images presented here are much deeper and have a higher resolution. We show mosaics of four CAFOS images (exposure time $20 \mathrm{~min}$ each, field size $\approx 16^{\prime}$ ). The resulting mosaic field size is $\approx 28^{\prime}$ (Fig. 1 ).

On our images MWP 1 appears to be an elliptical PN with axis lengths of $14^{\prime} 45^{\prime \prime}$ and $8^{\prime} 36^{\prime \prime}$ (Fig. 1 ). At a distance of $1.4_{-0.5}^{+0.7} \mathrm{kpc}$ (Motch et al. 1993) its linear diameter is $6_{-2}^{+3} \mathrm{pc}$.

\footnotetext{
${ }^{2}$ Röntensatellit.
} 


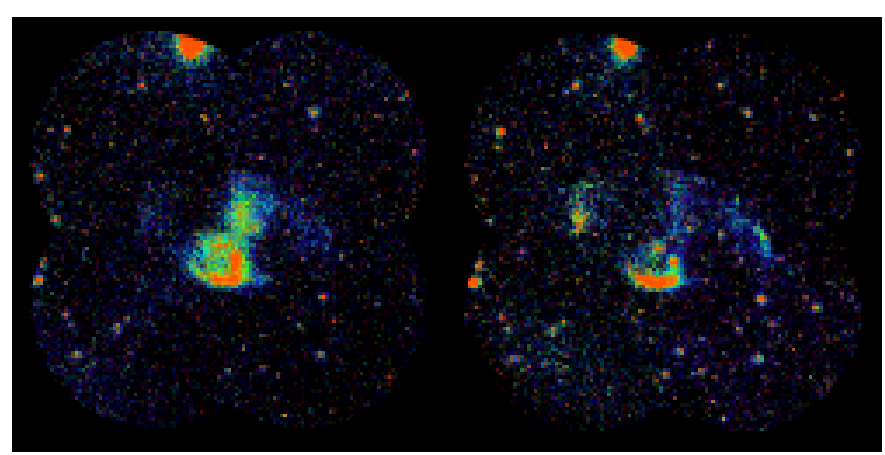

Fig. 1. MWP 1. The $\mathrm{H} \alpha$ image is shown left, [O III $\lambda$ $5007 \AA$ right

With its tremendous size, MWP 1 is one of the largest known PN: Two other PN, IsWe 2 (PN G107.7+07.8, $d=$ $6.5 \mathrm{pc})$ and Sh $2-176$ (PN G120.2-17.6, > $7 \mathrm{pc}$ ), have similar extensions but their diameters have been calculated using the strength of the interstellar NaD lines in order to determine their distance (Napiwotzki \& Schönberner 1995) which entails a relatively large error limit.

The large PN MWP1, however, is a challenge for evolutionary theory because its expansion time (about 150000 years if we assume $v_{\text {exp }}=20 \mathrm{~km} \mathrm{~s}^{-1}$ ) is at least two orders of magnitude longer than the time since the central star's departure from the AGB (about 1000 years, Blöcker 1995). One possible explanation for this discrepancy may be found in the born-again post-AGB nature (Iben et al. 1983) of the central star: A late He-shell flash at already declining luminosity brings back the star to the AGB and it experiences a second, He-burning, postAGB phase on a three times longer time scale. However, a "normal" $\mathrm{PN}$ with $M_{\mathrm{PN}}=0.2 M_{\odot}$ and an expansion velocity of $v_{\exp }=20 \mathrm{~km} \mathrm{~s}^{-1}$ will disperse in about 20000 years below the detection limit and hence, MWP 1 has to have extraordinary parameters. Nebula spectra with a high $\mathrm{S} / \mathrm{N}$ ratio as well as measurements of the expansion velocity are required in order to investigate this problem.

\section{MeWe 1-5, MeWe 1-8, and MeWe 1-9}

The three PNe MeWe 1-5, MeWe 1-8, and MeWe 1-9 were found by Melmer \& Weinberger (1990) by an inspection of ESO R / SERC J films. They have been verified to be true PNe by spectra taken with the ESO B\&C spectrograph (see Acker et al. 1992).

On our images, MeWe 1-5 appears just above the background with a faint arc on the western side (Fig. 2). MeWe 1-8 (Fig. 3) and MeWe 1-9 (Fig. 4) are circular PNe with no distinct central hole.

Although the central star of MeWe 1-5 has a brightness $m_{B}=20.6$ (Melmer \& Weinberger 1990) which is not much fainter than that of the central star of MeWe 1-8 $\left(m_{B}=20.3\right)$, it can, in contrast, not be identified on our

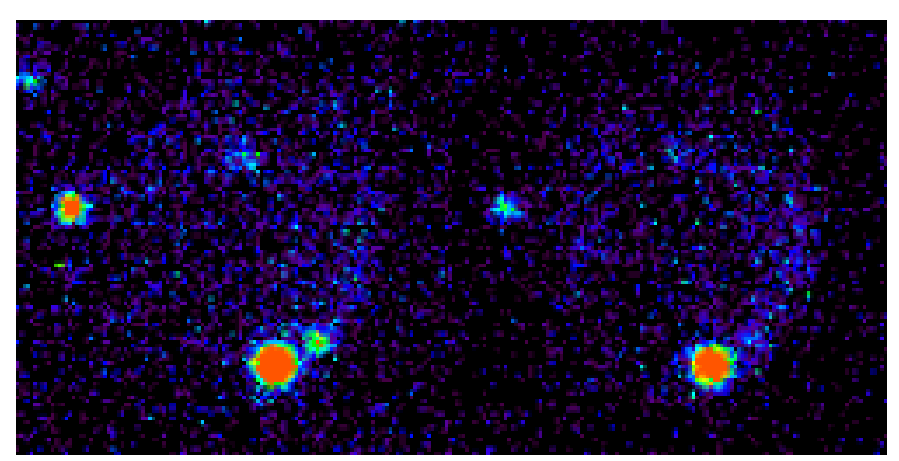

Fig. 2. MeWe 1-5. The PN is very faint and appears just above the background. Its central star cannot be identified

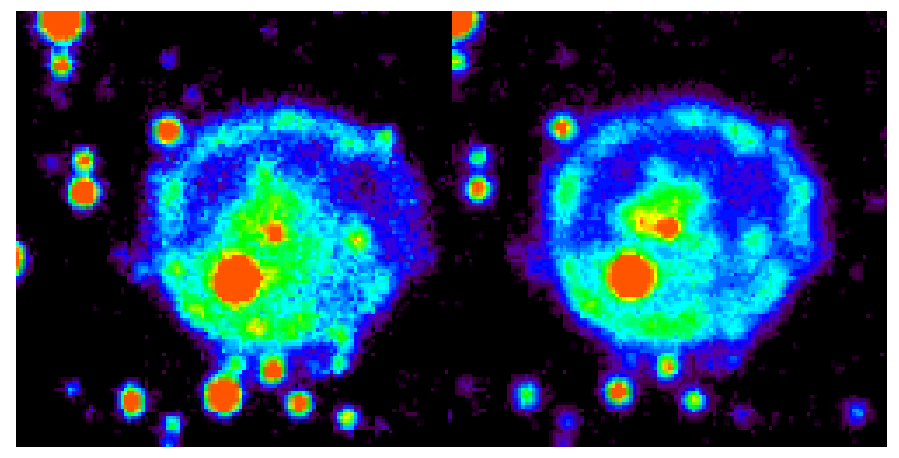

Fig. 3. MeWe 1-8

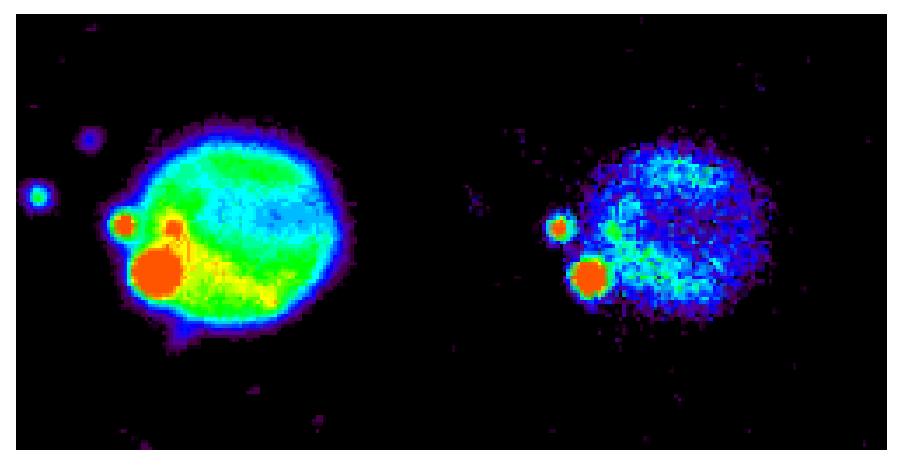

Fig. 4. MeWe 1-9. Note that there is no prominent central star

narrow-band images. In the case of MeWe 1-9, we cannot identify its central star $\left(m_{B}=21.9\right)$, too.

\section{A 43 and NGC 7094}

A 43 has been discovered by Abell (1955), NGC 7094 by Kohoutek (1963). They have been classified as Es- (elliptical, with inner structures) and Rs-type (round, with inner structures) PN, respectively, by Manchado et al. (1996).

The central stars of A 43 and NGC 7094 are so-called "hybrid" PG 1159 stars (Napiwotzki 1992; Werner 1992) which have a significant amount of hydrogen in their photospheres which shows that there is no sharp delimitation between the $\mathrm{H}$-normal and $\mathrm{H}$-deficient post-AGB stars (cf. Werner et al. 1997). 


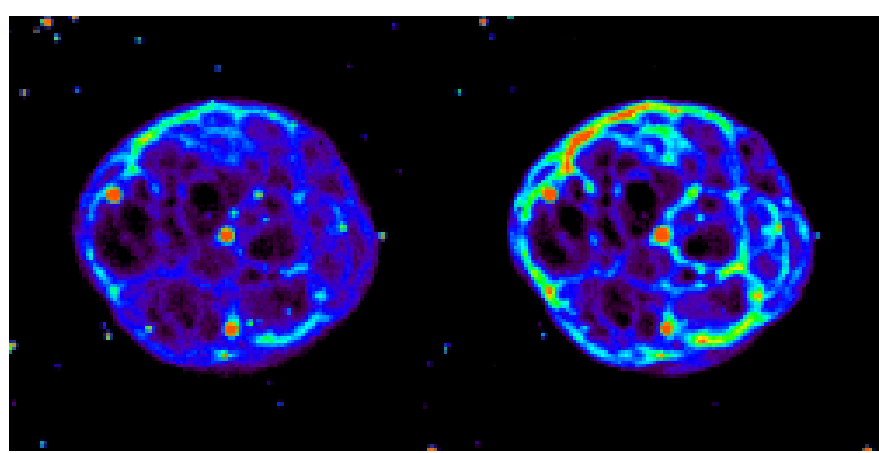

Fig. 5. A 43. The PN appears almost circular with filament-like structures

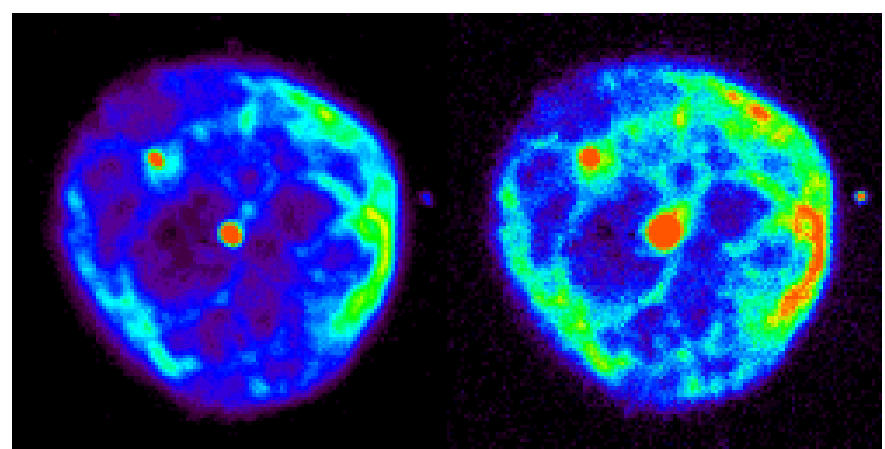

Fig. 6. NGC 7094. The PN is apparently similar to A 43 (Fig. 5) but, in contrast, the extension of its shell is about $10 \%$ smaller towards the SE and thus, not perfectly circular

At first glance, one can have the impression to see "Galactic footballs" (Figs. 5, 6), i.e. a kind of spherical shell with denser matter at the "seams" where the pentagons and hexagons meet - a foot(soccer)ball is made out of 12 pentagons and 20 hexagons (Kroto et al. 1985). The penta- and hexagons are more prominent in Fig. 5. However, this interpretation is somewhat subjective, e.g. Dwarkadas \& Balick (1998) mentioned A 43 with "filamentary structure with radial orientation", NGC 7094 with "prominent filamentary structure at the surface". Velocity measurements taken in order to derive the distribution of nebula matter in the line of sight would constrain a deprojection and help to achieve a realistic picture in this case.

The most likely explanation for the instabilities in these nebulae's surfaces might be that the old, slow AGB wind matter is swept up to a thin shell by the fast central star wind. While the invisible inner, high-pressure bubble is expanding due to the released energy of the stellar wind, instabilities in the dense, moving shell may appear (Vishniac 1983), effective enough to produce filament-like surface structures of the shell matter. As these filaments form, the intrafilament region can expand out ahead of the filaments, giving rise to a somewhat "lumpy" outer edge on the shell. This is quite obvious on the image of Abell 43 (Fig. 5).

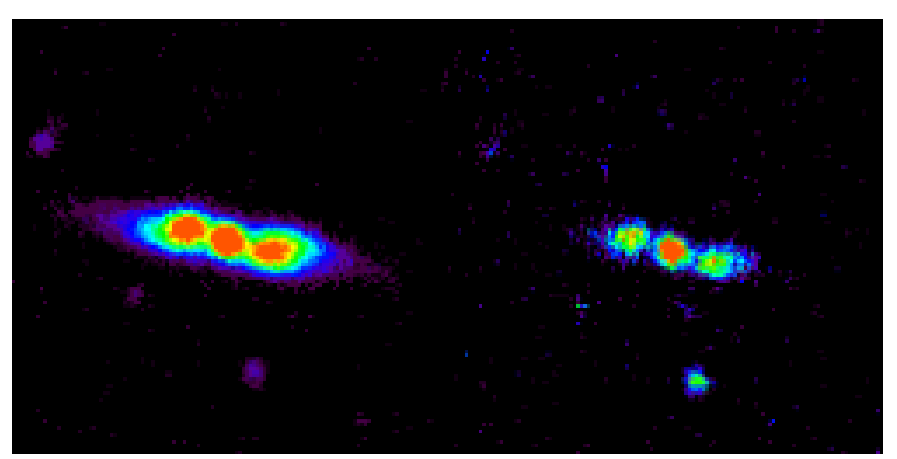

Fig. 7. He 2-437. This $\mathrm{PN}$ is highly bipolar. The inner nebula appears much brighter than the outer "fans" in [O III $] \lambda 5007 \AA$ which indicates different nebula properties. This deserves a detailed spectroscopic analysis

Similar PNe are known, e.g. NGC 6894, NGC 7048, or NGC 7139 (Balick 1987) but the edges of their shells appear smooth and round in projection. Thus, Abell 43 is an excellent test case for hydrodynamical simulations!

In any case, a reliable attempt to explain the morphologies of A 43 and NGC 7094 and the mentioned instabilities requires a detailed kinematic and spectroscopic study along with three-dimensional (magneto-) hydrodynamical models which account for the properties of the stellar wind.

\section{He 2-437}

He 2-437 is a PN which was verified by Henize (1967). His interpretation of the Palomar Atlas images was "a bright star between teardrop-shaped fans of nebulosity". On our images we see a bipolar nebular with a smaller but brighter circular nebula in the middle (Fig. 7). From the images it appears that the excitation in the middle part of the nebula is higher.

We have taken low-resolution spectra which verify the nebular origin of the emission. The shape of He 2-437 reminds of a napkin with a ring.

\section{NGC 1360}

NGC 1360 is an elliptical PN (Fig. 8) which was discovered by Minkowski (1946). It has an asymmetrical intensity distribution in the inner part with a maximum at the North (Fig. 8).

NGC 1360 has an apparent angular size of about $450^{\prime \prime} \times$ $360^{\prime \prime}$ in $\mathrm{H} \alpha$ and about $540^{\prime \prime} \times 430^{\prime \prime}$ in $[\mathrm{O}$ iII $] \lambda 5007 \AA$. At a HIPPARCOS distance of $350 \mathrm{pc}$ (Acker et al. 1998) its linear size is about $0.46 \times 0.36 \mathrm{pc}$. Given an [O III] expansion velocity of $v_{\exp }=28 \mathrm{~km} \mathrm{~s}^{-1}$ (Sabbadin 1984), the expansion time of the $\mathrm{PN}$ can be estimated from its largest extension to be about 16000 years.

A close look onto the PN images shows some features which look like turbulences towards the SE. Moreover, 


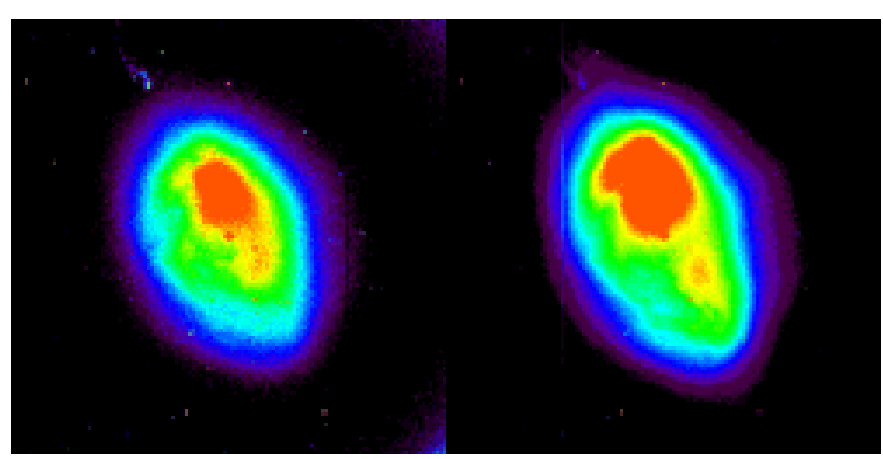

Fig. 8. NGC 1360. The PN appears larger in [O III $] \lambda 5007 \AA$ (right). Note that probably so-called "FLIERs" are found in the NE and the SW

FLIERs $^{3}$ (cf. Dwarkadas \& Balick 1998) are visible outside the main nebula along its axis (Fig. 8).

As interesting as PN NGC 1360 is its exciting star: It is one out of four extremely hot "H-normal" CSPN (the others are the CSPNe NGC 6853, NGC 4361, and NGC 3587). All have been detected by $\mathrm{EUVE}^{4}$, the CSPNe NGC 1360 and NGC 6853, were detected by ROSAT, too. Since the CSPN NGC 1360 is the brightest of this group, it is of considerable interest for testing NLTE models. NLTE model atmosphere analyses (Hoare et al. 1996) revealed $T_{\text {eff }}=110 \mathrm{kK}$ and $\log g=6$.

\section{Conclusions}

From our images we conclude that it appears worth doing systematically further very deep ground-based imaging of PN at high angular resolution in order to study their morphology in detail. Interesting information about the ejection mechanism of the nebula and its interaction with the ISM can be achieved. Moreover, detailed data about the spatial distribution of matter is a prerequisite for the use of deprojection techniques (Bremer 1995) which will allow to check nebula models against three-dimensional (magneto-)hydrodynamical calculations.

The search for PN around 27 objects (Werner et al. 1997 and this work) has shown only one possible candidate. This leaves the problem of "missing" PN around stars in the post-AGB region (see Rauch et al. 1998) unsolved.

Acknowledgements. We like to thank Bruce Balick for helpful comments on the morphology of Abell 43 and NGC 1360, Florian Kerber and Stefan Kimeswenger who supplied targets for our observing campaign, and Jörn Wilms for his assistance in IDL artwork. This research has made use of the SIMBAD database, operated at CDS, Strasbourg, France. This work was supported by use of the Data Base of Galactic Planetary

\footnotetext{
${ }^{3}$ Fast Low Ionisation Emission Regions.

${ }^{4}$ Extreme Ultraviolet Explorer.
}

Nebulae (www: http://ast2.uibk.ac.at) at Innsbruck. This research was supported by the Deutsches Zentrum für Luft- und Raumfahrt (DLR) under grant 50 OR 97055.

\section{References}

Abell G.O., 1955, PASP 67, 258

Acker A., Ochsenbein F., Tylenda R., Marcont J., Schohn C., 1992, Strasbourg-ESO Catalogue of galactic planetary nebulae

Acker A., Fresneau A., Pottasch S.R., Jasniewicz G., 1998, A\&A 337, 253

Appleton P.N., Kawaler S.D., Eitter J.J., 1993, AJ 106, 1973

Balick B., 1987, AJ 94, 971

Balick B., Alexander J., Hajian A., et al., 1998, AJ 116, 360

Blöcker T., 1995, A\&A 299, 755

Bremer M., 1995, A\&AS 112, 551

Dreizler S., Heber U., 1998, A\&A 334, 618

Dreizler S., Werner K., 1996, A\&A 314, 217

Dreizler S., Werner K., 1997, in: Isern J., Hernanz M., GarciaBerro E. (eds.). White Dwarfs, Kluwer, Dordrecht, p. 213

Dwarkadas V.V., Balick B., 1998, ApJ 497, 267

Henize K.G., 1967, ApJS 14, 125

Hoare M.G., Drake J.J., Werner K., Dreizler S., 1996, MNRAS 283,830

Iben I.Jr., Kaler J.B., Truran J.W., Renzini A., 1983, ApJ 264, 605

Kohoutek L., 1963, Bull. Astron. Inst. Czech. 13, 120

Kroto H.W., Heath J.R., O'Brien S.C., Curl R.F., Smalley R.E., 1985, Nat 318, 162

Manchado A., Guerrero M.A., Stanghellini L., Serra-Ricart M. 1996, The IAC Morphological Catalog of Northern Galactic Planetary Nebulae

Melmer D., Weinberger R., 1990, MNRAS 243, 236

Minkowski R., 1946, PASP 58, 305

Motch C., Belloni T., Buckley D., et al., 1991, A\&A 246, 24

Motch C., Werner K., Pakull M.W., 1993, A\&A 268, 561

Napiwotzki R., 1992, in: Heber U., Jeffery C.S. (eds.) Atmospheres of Early-Type Stars, Lecture Notes in Physics 401. Springer, Berlin, p. 310

Napiwotzki R., Schönberner D., 1995, A\&A 301, 545

Rauch T., Werner K., 1998, in: Philip A.G.D., Liebert J., Saffer R.A. (eds.) The Third Conference on Faint Blue Stars. L. Davis Press, Schenectady, NY, p. 217

Rauch T., Köppen J., Werner K., 1994, A\&A 286, 543

Rauch T., Köppen J., Werner K., 1996, A\&A 310, 613

Rauch T., Dreizler S., Wolff B., 1998, A\&A 338, 651

Sabbadin F., 1984, A\&AS 58, 273

Soker N., Dgani R., 1997, ApJ 484, 277

Tody D., 1986, in: Crawford D.L. (ed.) Proc. SPIE 627, 733

Tweedy R.W., Kwitter K.B., 1996, ApJ 107, 255

Vishniac E.T., 1983, ApJ 274, 152

Werner K., 1992, in: Heber U., Jeffery C.S. (eds.) Atmospheres of Early-Type Stars, Lecture Notes in Physics 401. Springer, Berlin, p. 273

Werner K., Dreizler S., Heber U., et al., 1996, A\&A 307, 860

Werner K., Dreizler S., Heber U., et al., 1997, in: Schielicke R.E. (ed.) Reviews in Modern Astronomy 10. Astronomische Gesellschaft, Hamburg, p. 219

Werner K., Bagschik K, Rauch T., Napiwotzki R., 1997, A\&A 327,721 